\title{
Extension of The Best Approximation Operator in Orlicz Spaces
}

\author{
Ivana Carrizo, Sergio Favier, and Felipe Zó \\ Instituto de Matemática Aplicada San Luis, UNSL-CONICET, Avda, Ejército de los Andes 950, \\ 5700 San Luis, Argentina
}

Correspondence should be addressed to Sergio Favier, sfavier@unsl.edu.ar

Received 17 December 2007; Accepted 26 February 2008

Recommended by Jean-Pierre Gossez

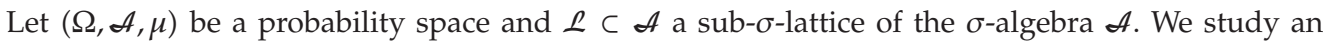
extension of the best $\phi$-approximation operator from an Orlicz space $L^{\phi}$ to the space $L^{\phi^{\prime}}$, where $\phi^{\prime}$ denotes the derivative of the convex, but not necessarily a strictly convex function $\phi$. We obtain convergence results when a sequence of $\sigma$-algebras $B_{n}$ converges to $B_{\infty}$ in a suitable way.

Copyright ( 92008 Ivana Carrizo et al. This is an open access article distributed under the Creative Commons Attribution License, which permits unrestricted use, distribution, and reproduction in any medium, provided the original work is properly cited.

\section{Introduction and notations}

Let $(\Omega, \mathcal{A}, \mu)$ be a probability space and let $\mathcal{M}=\mathcal{M}(\Omega, \mathcal{A}, \mu)$ be the set of all $\mathscr{A}$-measurable real-valued functions defined on $\Omega$. Given a $C^{1}$ convex function $\phi:[0, \infty) \rightarrow[0, \infty)$ such that $\phi(0)=0, \phi(t)>0$, when $t>0$, let $L^{\phi}=L^{\phi}(\Omega, \mathcal{A}, \mu)$ be the space of all functions $f \in \mathcal{M}$ such that

$$
\int_{\Omega} \phi(\lambda|f|) d \mu<\infty
$$

for some $\lambda>0$. Since we only deal with a $\Delta_{2}$ function $\phi$, that is, there exists a constant $K>0$ such that $\phi(2 x) \leq K \phi(x)$ for all $x \geq 0$, the space $L^{\phi}$ can be defined as the space of all function $f \in \mathcal{M}$, where (1.1) holds for every positive number $\lambda$. The space $L^{\phi^{\prime}}$ is analogously defined, where $\phi^{\prime}$ is the derivative of the function $\phi$. Besides, observe that for a $\Delta_{2}$ function $\phi$ it holds the next inequality

$$
\phi(x) \leq x \phi^{\prime}(x) \leq \phi(2 x) \leq K \phi(x)
$$

for all $x \geq 0$, and therefore $L^{\phi} \subset L^{\phi^{\prime}}$. Moreover $\varphi$ is a $\Delta_{2}$ function if and only if $\varphi^{\prime}$ is a $\Delta_{2}$ function. 
We say, according to [1], that a collection $\mathcal{L}$ of sets in $\mathcal{A}$ is a $\sigma$-lattice if it is closed under countable unions and intersections and contains $\varnothing$ and $\Omega$. Given a $\sigma$-lattice $\mathcal{L}$, we denote by $\overline{\mathcal{L}}$ the $\sigma$-lattice of all the complementary sets of $\mathcal{L}$, that is, $\overline{\mathcal{L}}=\left\{A^{c}: A \in \mathcal{L}\right\}$. Denote by $L^{\phi}(\mathcal{L})$ all $\mathcal{L}$-measurable functions in $L^{\phi}$.

A set $C \subset L^{\phi}$ is called $\phi$-closed if and only if $f_{n} \in C$ and $f_{n} \nearrow f \in L^{\phi}$ or $\left(f_{n} \searrow f \in L^{\phi}\right)$ then $f \in C$. Then $L^{\phi}(\mathcal{L})$ is a $\phi$-closed convex set and a lattice, that is closed for the maximum and minimum of functions.

We will use the notation $f \wedge g=\min (f, g)$ and $f \vee g=\max (f, g)$. A set $C \subset L^{\phi}$ is called a $\sigma$-complete lattice if and only if $\bigwedge_{n \in \mathbb{N}} f_{n}=\inf _{n \in \mathbb{N}} f_{n} \in C$ and $\bigvee_{n \in \mathbb{N}} f_{n}=\sup _{n \in \mathbb{N}} f_{n} \in C$ for all sequence $\left\{f_{n}\right\}_{n \in \mathbb{N}} \subset C$.

It is well known, see [1], that for every $f \in L^{\phi}$ there exists an element $g \in L^{\phi}(\mathcal{L})$ such that

$$
\int_{\Omega} \phi(|f-g|) d \mu=\inf _{h \in L^{\phi}(\mathcal{L})} \int_{\Omega} \phi(|f-h|) d \mu .
$$

Denote by $\mu_{\phi}^{\mathcal{\rho}}(f)$ the set of all $g \in L^{\phi}(\mathcal{L})$ satisfying (1.3). Each element of $\mu_{\phi}^{\mathcal{\rho}}(f)$ will be called a best $\phi$-approximation of $f$ given $L^{\phi}(\mathcal{L})$, and we will refer to the mapping $f \rightarrow \mu_{\phi}^{\mathcal{\rho}}(f)$ defined on $L^{\varphi}$ as the best approximation operator.

It is showed in [1] that for $f \in L^{\phi}$ the set $\mu_{\phi}^{\mathcal{L}}(f)$ is a nonvoid $\sigma$-complete lattice. Also it was proved that if $f \leq g$, both in $L^{\phi}, f_{1} \in \mu_{\phi}^{\rho}(f)$ and $g_{1} \in \mu_{\phi}^{\rho}(g)$ then we have $f_{1} \wedge f_{2} \in \mu_{\phi}^{\rho}(f)$ and $f_{1} \vee f_{2} \in \mu_{\phi}^{\mathcal{\rho}}(g)$. In this case, we say that the multivalued operator $\mu_{\phi}^{\mathcal{\rho}}(f)$ is a monotone operator.

The main purpose of this paper is to extend the best approximation operator to the set $L^{\varphi^{\prime}}$. The case $\varphi(t)=t^{p}, p>1$, was extensively treated in [2] and the best $L^{1}$ approximation operator is extended to all measurable functions in [3]. The extension from $L^{\varphi}$ to $L^{\varphi^{\prime}}$ is considered in [4] for a $C^{1}$ function $\varphi$ which is strictly convex and $\varphi^{\prime}(0)=0$.

Now in this paper we consider a $C^{1}$ convex function $\phi, \phi^{\prime}(0)=0$, but not necessarily a strictly convex function. Extension of best approximation operator when the approximation classes are the constants is treated in [5-7].

The extension of the best approximation operator is $\mu_{\phi}^{\mathcal{\rho}}(f)$; for $f \in L^{\phi^{\prime}}$, we will be denoted by $\tilde{\mu}_{\phi}^{\mathcal{\rho}}(f)$. In Theorem 2.12, we prove that $\tilde{\mu}_{\phi}^{\mathcal{L}}(f) \neq \varnothing$ for every $f \in L^{\phi^{\prime}}$, while in Theorem 2.16 it is proved that it is indeed an extension, that is, $\tilde{\mu}_{\phi}^{\mathcal{\rho}}(f)=\mu_{\phi}^{\mathcal{\rho}}(f)$ for $f \in L^{\phi}$. Additional properties are obtained for the set $\tilde{\mu}_{\phi}^{\mathcal{\rho}}(f)$ when the $\sigma$-lattice $\mathcal{L}$ is a $\sigma$-algebra (see Theorem 2.17) and similar results hold when $\mathcal{L}$ is the class of monotone functions in $L^{\phi^{\prime}}$ (see Theorem 3.2). A martingale-type result is given in Theorem 4.1 which generalizes [8, Theorem 2.8 ] for the particular family of measures considered in this paper.

\section{Extension of the best $\phi$-approximation operator}

We begin with some definitions and auxiliary results. The proof of the next two lemmas can be found in [4].

Lemma 2.1. A necessary and sufficient condition for a continuously differentiable and convex function $\phi$ to satisfy the $\Delta_{2}$ condition is that there exists a constant $\alpha>1$ such that

$$
u \phi^{\prime}(u) \leq \alpha \phi(u) \quad \forall u \geq 0 .
$$


Lemma 2.2. Let $f$, $g$ be in $L^{\phi}$. Then $\phi^{\prime}(|f|) g$ is an integrable function.

According to Brunk and Johansen [8], we set the following definitions.

Definition 2.3. Let $v$ be a signed measure on $\mathcal{A}$ and let $\mathcal{L}$ be a $\sigma$-lattice contained in $\mathcal{A}$. Say that $P \in \mathcal{L}$ is a $v$-positive set, if for all $D \in \overline{\mathcal{L}}$, then $\mathcal{v}(P \cap D) \geq 0$. A set $N \in \overline{\mathcal{L}}$ is called $v$-negative if for all $C \in \mathcal{L}$ one has $v(N \cap C) \leq 0$.

Definition 2.4. Let $\left\{v_{a}\right\}_{a \in \mathbb{R}}$ be a family of measures on $\mathcal{A}$, and let $\mathcal{L}$ be a $\sigma$-lattice contained in $\mathcal{A}$. An $\mathcal{L}$-measurable function $g$ is called a Lebesgue-Radon-Nikodym function (LRN function) for $\left\{v_{a}\right\}$ given $\mathcal{L}$ if and only if the set $\{g>a\}$ is $v_{a}$-positive for all $a \in \mathbb{R}$ and the set $\{g<a\}$ is $v_{a}$-negative for all $a \in \mathbb{R}$.

Remark 2.5. We note that in Definition 2.4 it is sufficient to impose the conditions for all $a$ in a dense set in $\mathbb{R}$, see [8, page 588].

For $f \in L^{\phi}, g \in L^{\phi}(\mathcal{L})$, and $a \in \mathbb{R}$, we define the following measures on $\mathcal{A}$ :

$$
\mu_{g}(A)=\int_{A} \underline{\phi}^{\prime}(f-g) d \mu, \quad \mu_{a}(A)=\int_{A} \underline{\phi}^{\prime}(f-a) d \mu,
$$

where $\phi^{\prime}(x)=\phi^{\prime}(|x|) \operatorname{sign}(x)$. Note that when $f \in L^{\phi^{\prime}}$ and $g \in L^{\phi^{\prime}}(\mathcal{L})$, the measure $\mu_{g}$ and $\mu_{a}$ are well defined.

The next theorem is a characterization of $\mu_{\phi}^{\rho}(f)$, see [9, Theorem 3.2].

Theorem 2.6. Let $f \in L^{\phi}, \mathcal{L} \subset \mathcal{A}$ be a $\sigma$-lattice and $g \in L^{\phi}(\mathcal{L})$. Then the following statement are equivalent.

(1) $g \in \mu_{\phi}^{\rho}(f)$.

(2) (a) a set $\{g>a\}$ is $\mu_{g}$-positive for all $a \in \mathbb{R}$; and

(b) a set $\{g<a\}$ is $\mu_{g}$-negative for all $a \in \mathbb{R}$.

(3) $g$ is an LRN function for the family $\left\{\mu_{a}\right\}_{a \in \mathbb{R}}$ given $\mathcal{L}$.

Now we extend the operator $\mu_{\phi}^{\mathcal{L}}(\cdot)$ to the space $L^{\phi^{\prime}}$.

Definition 2.7. Let $\mathcal{L}$ be a $\sigma$-lattice and let $f \in L^{\phi^{\prime}}$. Then $g$ is an extended best $\phi$-approximation if and only if $g \in L^{\phi^{\prime}}(\mathcal{L})$ and

(i) the set $\{g>a\}$ is $\mu_{g}$-positive for all $a \in \mathbb{R}$;

(ii) the set $\{g<a\}$ is $\mu_{g}$-negative for all $a \in \mathbb{R}$.

For $f \in L^{\phi^{\prime}}$ we denote by $\tilde{\mu}_{\phi}^{\mathcal{\rho}}(f)$ the set of all extended best $\phi$-approximation functions.

Remark 2.8. Let $f \in L^{\phi^{\prime}}$ and let $g$ be a function in $L^{\phi^{\prime}}(\mathcal{L})$ such that the set $\{g>a\}$ is $\mu_{g}$-positive and the set $\{g<a\}$ is $\mu_{g}$-negative for all $a \in \mathbb{R}$. Then we have the following.

(i) For all $h \in L^{\infty}(\overline{\mathcal{L}})$ and $h \geq 0$,

$$
\int_{\{g>a\}} \underline{\phi}^{\prime}(f-g) h d \mu \geq 0 .
$$


For all $h \in L^{\infty}(\mathcal{})$ and $h \geq 0$,

$$
\int_{\{g<a\}} \underline{\phi}^{\prime}(f-g) h d \mu \leq 0
$$

(ii)

$$
\int \underline{\phi^{\prime}}(f-g) d \mu=0
$$

Proof. We prove inequality (2.3). Since the set $\{g>a\}$ is $\mu_{g}$-positive, that is, for each $D \in \overline{\mathcal{L}}$ and $a \in \mathbb{R}$, we have

$$
\int_{\{g>a\} \cap D} \underline{\phi^{\prime}}(f-g) d \mu \geq 0
$$

For $h=\sum_{k=1}^{N} c_{k} X_{D_{k}}$, where $D_{k} \in \overline{\mathcal{L}}$ and $c_{k} \geq 0, k=1, \ldots, N$, then by (2.6), we have

$$
\int_{\{g>a\}} \underline{\phi}^{\prime}(f-g) h d \mu=\sum_{k=1}^{N} c_{k} \int_{\{g>a\} \cap D_{k}} \underline{\phi}^{\prime}(f-g) d \mu \geq 0 .
$$

All nonnegative $h \in L^{\infty}(\overline{\mathcal{L}})$ can be obtained as a limit of functions of the above type. The proof of inequality (2.4) is similar.

The equality (2.5) is obtained using Lebesgue's theorem when $a \rightarrow \infty$ with $h=1$ in (2.4) and if $a \rightarrow-\infty$ consider in (2.3) also $h=1$.

As a reference, we note that (2) is equivalent to (3) in Theorem 2.6 for $f \in L^{\phi^{\prime}}, g \in L^{\phi^{\prime}}$. We have the next remark.

Remark 2.9. For $f \in L^{\phi^{\prime}}$ and $g \in L^{\phi^{\prime}}(\mathcal{})$, the following statements are equivalent:

(1) $g \in \tilde{\mu}_{\phi}^{\rho}(f)$;

(2) $g$ is an LRN function for the family $\left\{\mu_{a}\right\}_{a \in \mathbb{R}}$ given $\mathcal{L}$.

The next lemma is a particular case of [8, Theorem 1.8].

Lemma 2.10. Let $f \in L^{\phi^{\prime}}$ and $g \in L^{\phi^{\prime}}(\mathcal{\perp})$. Then the following statements are equivalent.

(1) $g$ is an LRN function for the family $\left\{\mu_{a}\right\}_{a \in \mathbb{R}}$ given $\mathcal{L}$.

(2) There exists a countable set $D$ such that $\{g \leq a\}$ is $\mu_{a}$-negative for all $a \in D^{c}$ and the set $\{g \geq a\}$ is $\mu_{a}$-positive for all $a \in D^{c}$.

We need the following auxiliary result.

Lemma 2.11. Let $f_{n} \in L^{\phi^{\prime}}$ be a sequence of functions such that $f_{n} \nearrow f\left(f_{n} \searrow f\right)$, where $f \in L^{\phi^{\prime}}$. Let $g_{n} \in \tilde{\mu}_{\phi}^{\perp}\left(f_{n}\right)$ be such that $g_{n} \nearrow g\left(g_{n} \searrow g\right)$. Then $g \in \tilde{\mu}_{\phi}^{\perp}(f)$. 
Proof. We will prove the result just for the increasing case, the proof for the decreasing case follows the same pattern. The function $g=\lim _{n \rightarrow \infty} g_{n}$ is obviously $\mathcal{L}$-measurable function. Now, we prove that $g \in L^{\phi^{\prime}}$, and it satisfies (i) and (ii) of Definition 2.7. We have that

$$
f_{n}-g_{n} \leq f-g_{n} \leq f-g_{1} .
$$

Using (2.5),

$$
\int_{\Omega} \underline{\phi^{\prime}}\left(f_{n}-g_{n}\right) d \mu=0 \quad \text { for each } n \in \mathbb{N} .
$$

According to (2.8) and (2.9), we have, since $\underline{\phi}^{\prime}(x)$ is an increasing function,

$$
\int_{\Omega} \underline{\phi}^{\prime}\left(f-g_{n}\right) d \mu \geq 0
$$

Since $\phi^{\prime}$ is a continuous function and $\phi^{\prime}(0)=0$, we have by (2.8) and Lebesgue's theorem

$$
\int_{\Omega}\left(\underline{\phi}^{\prime}(f-g) \vee 0\right) d \mu \leq \int_{\Omega}\left(\underline{\phi}^{\prime}\left(f-g_{1}\right) \vee 0\right) d \mu .
$$

Now by (2.10) it holds

$$
\int_{\Omega}\left(-\underline{\phi}^{\prime}\left(f-g_{n}\right) \vee 0\right) d \mu \leq \int_{\Omega}\left(\underline{\phi}^{\prime}\left(f-g_{n}\right) \vee 0\right) d \mu .
$$

Using Fatou in (2.12), we have

$$
\int_{\Omega}\left(-\underline{\phi}^{\prime}(f-g) \vee 0\right) d \mu \leq \int_{\Omega}\left(\underline{\phi}^{\prime}(f-g) \vee 0\right) d \mu .
$$

Therefore, using (2.11) and (2.12), we get $g \in L^{\phi^{\prime}}(\Omega)$ and $\int_{\Omega} \underline{\phi^{\prime}}(f-g) d \mu \geq 0$.

Let $a \in \mathbb{R}, D \in \overline{\mathcal{L}}$, and $C \in \mathcal{L}$ we know for each $n$

$$
\int_{\left\{g_{n}>a\right\} \cap D} \underline{\phi^{\prime}}\left(f_{n}-g_{n}\right) d \mu \geq 0, \quad \int_{\left\{g_{n}<a\right\} \cap C} \underline{\phi}^{\prime}\left(f_{n}-g_{n}\right) d \mu \leq 0 .
$$

Since $\left\{g_{n}\right\}_{n \in \mathbb{N}}$ is an increasing sequence, we get $\bigcup_{n \in \mathbb{N}}\left\{g_{n}>a\right\}=\{g>a\}$, and by (2.14), we have

$$
\int_{\{g>a\} \cap D} \underline{\phi^{\prime}}(f-g) d \mu \geq 0 .
$$

Hence, the set $\{g>a\}$ is $\mu_{g}$-positive for all $a \in \mathbb{R}$.

Now, for $a \in \mathbb{R}$ and $k, n \in \mathbb{N}$, we define $B_{n}=\left\{g_{n}<a\right\}$ and $B_{n, k}=\left\{g_{n}<a-1 / k\right\}$. We have that, for $n \rightarrow \infty, B_{n, k} \searrow A_{k}$ for some $\mathcal{A}$-measurable set such that

$$
\left\{g<a-\frac{1}{k}\right\} \subset A_{k} \subset\left\{g \leq a-\frac{1}{k}\right\} .
$$


We observe that $\mathcal{X}_{A_{k}} \rightarrow \mathcal{X}_{\{g<a\}}$, a.e. Then taking limit as $k \rightarrow \infty, n \rightarrow \infty$ and using Lebesgue's theorem, we obtain

$$
\int_{\{g<a\} \cap C} \underline{\phi}^{\prime}(f-g) d \mu=\lim _{k \rightarrow \infty} \int \underline{\phi^{\prime}}(f-g) x_{A_{k} \cap C} d \mu=\lim _{k \rightarrow \infty} \lim _{n \rightarrow \infty} \int_{B_{n, k} \cap C} \underline{\phi}^{\prime}(f-g) d \mu \leq 0 .
$$

Theorem 2.12. Let $\mathcal{\perp}$ be a $\sigma$-lattice and $f \in L^{\phi^{\prime}}$, then $\tilde{\mu}_{\phi}^{\mathcal{L}}(f) \neq \varnothing$.

Proof. For $f \in L^{\phi^{\prime}}$ we can define the following sequences. For each $m \in \mathbb{N}$, let $f_{m}=f \vee(-m)$ and when $m \rightarrow \infty$ we have $f_{m} \searrow f$. Set $f_{m}^{n}=(f \vee(-m)) \wedge n$ for all $n, m$ in $\mathbb{N}$, then we have $f_{m n} \nearrow f_{m}$, when $n \rightarrow \infty$. Since for each $m, n \in \mathbb{N}$ we have $f_{m}^{n} \in L^{\phi}$, there exist $g_{m}^{n} \in \mu_{\phi}^{\rho}\left(f_{m}^{n}\right)$. As $\mu_{\phi}^{\rho}(\cdot)$ is a mono-tone operator over $L^{\phi}$ we can take a new sequence that we call again $g_{m}^{n} \in \mu_{\phi}^{\rho}\left(f_{m}^{n}\right)$, such that $g_{m}^{n} \leq g_{m}^{n+1}$ for all $n \in \mathbb{N}$.

Since $f_{m}^{n} \geq f_{m+1}^{n}$ and using again that $\mu_{\phi}^{\rho}(\cdot)$ is a monotone operator, we have $\widetilde{g}_{m+1}^{n} \leq \widetilde{g}_{m}^{n}$, where $\widetilde{g}_{m}^{n} \in \mu_{\phi}^{\rho}\left(f_{m}^{n}\right)$ is the sequence defined by $\widetilde{g}_{1}^{n}=g_{1}^{n}$ and $\widetilde{g}_{m+1}^{n}=\widetilde{g}_{m}^{n} \wedge g_{m+1}^{n}$. Furthermore, it is easy to check that $\widetilde{g}_{m}^{n} \leq \widetilde{g}_{m}^{n+1}$.

Then, we have that for each $m \in \mathbb{N}$ that $f_{m}^{n} \nearrow f_{m}$, when $n \rightarrow \infty$, and since $\mu_{\phi}^{\rho}\left(f_{m}^{n}\right) \subset$ $\tilde{\mu}_{\phi}^{\rho}\left(f_{m}^{n}\right)$ we have $\tilde{g}_{m}^{n} \in \tilde{\mu}_{\phi}^{\rho}\left(f_{m}^{n}\right)$ and if we define $g_{m}=\lim _{n \rightarrow \infty} \widetilde{g}_{m}^{n}$ by Lemma 2.11 we obtain $g_{m} \in \tilde{\mu}_{\phi}^{\mathcal{L}}\left(f_{m}\right)$ and $g_{m} \geq g_{m+1}$ for all $m \in \mathbb{N}$. If we take $m \rightarrow \infty$, we have $f_{m} \searrow f$ and by Lemma 2.11 we get $g \in \tilde{\mu}_{\phi}^{\rho}(f)$, where $g=\lim _{m \rightarrow \infty} g_{m}$.

To see that the extended best $\phi$-approximation is an extension of the best $\phi$-approximation operator, we must prove $\tilde{\mu}_{\phi}^{\rho}(f)=\mu_{\phi}^{\rho}(f)$ for every $f \in L^{\phi}$. First, we need to prove the following lemmas.

Lemma 2.13. Let $\phi$ be a $C^{1}$ convex function and assume that it satisfies the $\Delta_{2}$-condition. Then

$$
\phi(a)+\frac{K}{2} a \underline{\phi}^{\prime}(x-a) \leq \frac{K+2}{2} \phi(x),
$$

for $a, x \geq 0$, where $K$ is the constant for the $\Delta_{2}$ condition.

Proof. We consider two cases. First, we assume $0<x \leq a$. Since $\phi$ is $\Delta_{2}$-convex function, we have that $\phi(a)=\phi(a-x+x) \leq(K / 2)(\phi(a-x)+\phi(x))$. Using $x \phi^{\prime}(x) \geq \phi(x)$ for all $x$, we get

$$
\begin{aligned}
\phi(x)+a \phi^{\prime}(a-x) & =\phi(x)+(a-x+x) \phi^{\prime}(a-x) \\
& \geq \phi(x)+\phi(a-x)+x \phi^{\prime}(a-x) \\
& \geq \frac{2}{K} \phi(a)+x \phi^{\prime}(a-x) \geq \frac{2}{K} \phi(a) .
\end{aligned}
$$

Then we obtain

$$
\phi(a)-\frac{K}{2} a \phi^{\prime}(a-x) \leq \frac{K}{2} \phi(x) .
$$

For $0 \leq a<x$, we have

$$
\phi(a)+a \frac{K}{2} \phi^{\prime}(x-a) \leq \phi(a)+\frac{K}{2} \int_{x-a}^{x} \phi^{\prime}(t) d t \leq \phi(x)+\frac{K}{2} \phi(x)=\frac{K+2}{2} \phi(x) .
$$


Lemma 2.14. Let $f \in L^{\phi}$ and $g \in \tilde{\mu}_{\phi}^{\rho}(f)$, then

$$
\int_{\{g>0\}} \underline{\phi^{\prime}}(f-g) g d \mu \geq 0
$$

Proof. Since $\{g>a\}$ is $\mu_{g}$-positive for all $a \in \mathbb{R}$, then for all $D \in \overline{\mathcal{L}}$, we have that

$$
\int_{\{g>a\} \cap D} \underline{\phi}^{\prime}(f-g) d \mu \geq 0 .
$$

In particular, it holds that for all $a \in \mathbb{R}$,

$$
\int_{\{g>a\}} \underline{\phi}^{\prime}(f-g) d \mu \geq 0
$$

that is,

$$
\int_{\{g>a\} \cap\{f>g\}} \phi^{\prime}(|f-g|) d \mu \geq \int_{\{g>a\} \cap\{f \leq g\}} \phi^{\prime}(|f-g|) d \mu .
$$

Now, we have

$$
\int_{0}^{\infty} \int_{\{g>a\} \cap\{f>g\}} \phi^{\prime}(|f-g|) d \mu d a \geq \int_{0}^{\infty} \int_{\{g>a\} \cap\{f \leq g\}} \phi^{\prime}(|f-g|) d \mu d a .
$$

By the Fubini's theorem, we get

$$
\int_{\{g>0\}} \int_{0}^{g} \phi^{\prime}(|f-g|) x_{\{f>g\}} d a d \mu \geq \int_{\{g>0\}} \int_{0}^{g} \phi^{\prime}(|f-g|) x_{\{f \leq g\}} d a d \mu .
$$

Thus

$$
\int_{\{g>0\}} \phi^{\prime}(|f-g|) x_{\{f>g\}} g d \mu \geq \int_{\{g>0\}} \phi^{\prime}(|f-g|) x_{\{f \leq g\}} g d \mu .
$$

To see that inequality (2.22) is equivalent to (2.28) we will prove that $\phi^{\prime}(f-g) x_{\{f>g\}} g \in L^{1}(\{g>$ $0\})$. In fact

$$
\int_{\{g>0\}} \phi^{\prime}(f-g) x_{\{f>g\}} g d \mu \leq \int_{\{g>0\}} \phi^{\prime}(f-g) x_{\{f>g\}} f d \mu \leq \int_{\{g>0\}} \phi^{\prime}(f) f .
$$

Since $f \in L^{\phi}$ by Lemma 2.2, the last integral is finite.

The following properties of the set $\tilde{\mu}_{\phi}^{\mathcal{P}}(f)$ can be easily proved.

Proposition 2.15. Let $f \in L^{\phi^{\prime}}$, then

(1) $-\tilde{\mu}_{\phi}^{\bar{\rho}}(-f)=\tilde{\mu}_{\phi}^{\mathcal{\rho}}(f)$,

(2) $\tilde{\mu}_{\phi}^{\mathcal{\rho}}(f+t)=\tilde{\mu}_{\phi}^{\mathcal{L}}(f)+t$ for all $h \in \mathbb{R}$.

Now we prove that the operator $\tilde{\mu}_{\phi}^{\mathcal{\rho}}(f)$ is in fact an extension of the operator $\mu_{\phi}^{\ell}(f)$. 
Theorem 2.16. Let $f \in L^{\phi}$, then $\tilde{\mu}_{\phi}^{\mathcal{L}}(f)=\mu_{\phi}^{\mathcal{\rho}}(f)$.

Proof. For $f \in L^{\phi}$, we will prove only that $\tilde{\mu}_{\phi}^{\mathcal{L}}(f) \subset \mu_{\phi}^{\mathcal{L}}(f)$. The other inclusion follows from Theorem 2.6. Let $g \in \tilde{\mu}_{\phi}^{\rho}(f)$ and again using Theorem 2.6 it remains to prove that $g \in L^{\phi}$. Recall that $\phi(0)=0$, then

$$
\int_{\Omega} \phi(|g|) d \mu=\int_{\{g>0\}} \phi(g) d \mu+\int_{\{g<0\}} \phi(-g) d \mu .
$$

By Lemma 2.13 we obtain the following inequality:

$$
\int_{\{g>0\}} \phi(g) d \mu+\frac{K}{2} \int_{\{g>0\}} \underline{\phi^{\prime}}(|f|-g) g d \mu \leq \frac{K+2}{2} \int_{\{g>0\}} \phi(|f|) d \mu .
$$

Applying Lemma 2.14 and taking into account that $\underline{\phi}^{\prime}(x)$ is an increasing function, we get

$$
0 \leq \int_{\{g>0\}} \underline{\phi^{\prime}}(f-g) g d \mu \leq \int_{\{g>0\}} \underline{\phi^{\prime}}(|f|-g) g d \mu .
$$

Thus using (2.32) in (2.31), we have

$$
\int_{\{g>0\}} \phi(g) d \mu \leq \frac{K+2}{2} \int_{\Omega} \phi(|f|) d \mu .
$$

For the set $\{g<0\}$, again by Lemma 2.13, we obtain

$$
\int_{\{g<0\}} \phi(-g) d \mu+\frac{K}{2} \int_{\{g<0\}} \underline{\phi}^{\prime}(|f|+g)(-g) d \mu \leq \frac{K+2}{2} \int_{\{g<0\}} \phi(|f|) d \mu .
$$

Since $-f+g \leq|f|+g$, we have $\underline{\phi}^{\prime}(-f+g) \leq \underline{\phi}^{\prime}(|f|+g)$. Thus

$$
\int_{\{g<0\}} \underline{\phi}^{\prime}(-f+g)(-g) d \mu \leq \int_{\{g<0\}} \underline{\phi}^{\prime}(|f|+g)(-g) d \mu .
$$

By (1) in Proposition 2.15, $-g \in \widetilde{\mu}_{\phi}^{\bar{\rho}}(-f)$, and by Lemma 2.14, we have that

$$
\int_{\{g<0\}} \underline{\phi^{\prime}}(-f+g)(-g) d \mu \geq 0
$$

Therefore, $\int_{\{g<0\}} \phi(-g) d \mu \leq((K+2) / 2) \int \phi(|f|) d \mu$. By (2.33), we have

$$
\int_{\Omega} \phi(|g|) d \mu \leq \frac{K+2}{2} \int_{\Omega} \phi(|f|) d \mu
$$

and therefore $g \in L^{\phi}$. 
Now, if consider a $\sigma$-subalgebra $ß$ instead of a $\sigma$-lattice $\mathcal{L}$, the extended best $\phi$-approximation operator has the following properties.

Theorem 2.17. Let $f, f_{1}$, and $f_{2}$ be in $L^{\phi^{\prime}}$, if $\boldsymbol{B}$ is a sub- $\sigma$-algebra of the $\sigma$-algebra $A$, then the following hold.

(1) The set-valued function $\tilde{\mu}_{\phi}^{\mathcal{B}}(f)$ is a monotone operator.

(2) The set $\tilde{\mu}_{\phi}^{\mathcal{B}}(f)$ is a $\sigma$-complete lattice, and there exist $U_{\mathcal{B}}, V_{\mathcal{B}} \in \tilde{\mu}_{\phi}^{B}(f)$ such that $U_{\mathcal{B}} \leq g \leq V_{\bar{B}}$ a.e. for every $g \in \tilde{\mu}_{\phi}^{B}(f)$.

Proof. To prove (1), recall that this set-valued operator is monotone if $f_{1} \leq f_{2}$; then if $g_{1} \in \tilde{\mu}_{\phi}^{\mathcal{B}}\left(f_{1}\right)$ and $g_{2} \in \tilde{\mu}_{\phi}^{\mathcal{B}}\left(f_{2}\right)$, we have that $g_{1} \wedge g_{2} \in \tilde{\mu}_{\phi}^{\mathcal{B}}\left(f_{1}\right)$ and $g_{1} \vee g_{2} \in \tilde{\mu}_{\phi}^{\mathcal{B}}\left(f_{2}\right)$. Since $L^{\phi}(\mathbb{B})$ is a lattice, we know $g_{1} \wedge g_{2} \in L^{\phi}(\mathbb{B})$ and $g_{1} \vee g_{2} \in L^{\phi}(\mathbb{B})$. We will prove first that $g_{1} \wedge g_{2} \in \tilde{\mu}_{\phi}^{\mathcal{B}}\left(f_{1}\right)$. Set $\mu_{a}^{f_{i}}(A)=\int_{A} \underline{\phi^{\prime}}\left(f_{i}-a\right) d \mu$, where $a \in \mathbb{R}$ and $i=1,2$. We will see that $g_{1} \wedge g_{2}$ is an LRN function for the family of measures $\left\{\mu_{a}^{f_{1}}\right\}_{a \in \mathbb{R}}$ given $\boldsymbol{B}$. First, we will see that for each $a \in \mathbb{R}$ and for all $B \in B$, we have

$$
\mu_{a}^{f_{1}}\left(\left\{g_{1} \wedge g_{2}>a\right\} \cap B\right) \geq 0 .
$$

Since $\left\{g_{1} \wedge g_{2}>a\right\} \cap B=\left\{g_{1}>a\right\} \cap\left\{g_{2}>a\right\} \cap B$ and $\left\{g_{2}>a\right\} \cap B \in \mathbb{B}$ and using that $g_{1}$ is an LRN function of the family $\left\{\mu_{a}^{f_{1}}\right\}_{a \in \mathbb{R}^{\prime}}$ we obtain that for all $B \in \mathcal{B}$

$$
\int_{\left\{g_{1} \wedge g_{2}>a\right\} \cap B} \underline{\phi}^{\prime}\left(f_{1}-a\right) d \mu=\int_{\left\{g_{1}>a\right\} \cap\left\{g_{2}>a\right\} \cap B} \underline{\phi}^{\prime}\left(f_{1}-a\right) d \mu \geq 0 .
$$

Now, we see that $\left\{g_{1} \wedge g_{2}<a\right\}$ is $\mu_{a}^{f_{1}}$-negative for all $a \in \mathbb{R}$. For $B \in \mathbb{B}$, we have

$$
\left\{g_{1} \wedge g_{2}<a\right\} \cap B=\left(\left\{g_{1}<a\right\} \cap B\right) \cup\left(\left\{g_{1} \geq a\right\} \cap\left\{g_{2}<a\right\} \cap B\right) .
$$

Using $f_{1} \leq f_{2}$ and that $\underline{\phi}^{\prime}(\cdot)$ is a nondecreasing function, we obtain

$$
\begin{aligned}
\int_{\left\{g_{1} \wedge g_{2}<a\right\} \cap B} \underline{\phi}^{\prime}\left(f_{1}-a\right) d \mu & =\int_{\left\{g_{1}<a\right\} \cap B} \underline{\phi}^{\prime}\left(f_{1}-a\right) d \mu+\int_{\left\{g_{1} \geq a\right\} \cap\left\{g_{2}<a\right\} \cap B} \underline{\phi^{\prime}}\left(f_{1}-a\right) d \mu \\
& \leq \int_{\left\{g_{1}<a\right\} \cap B} \underline{\phi}^{\prime}\left(f_{1}-a\right) d \mu+\int_{\left\{g_{1} \geq a\right\} \cap\left\{g_{2}<a\right\} \cap B} \underline{\phi}^{\prime}\left(f_{2}-a\right) d \mu \leq 0 .
\end{aligned}
$$

Thus

$$
\int_{\left\{g_{1} \wedge g_{2}<a\right\} \cap B} \underline{\phi}^{\prime}\left(f_{1}-a\right) d \mu \leq 0 .
$$

By (2.39) and (2.42), we have $g_{1} \wedge g_{2} \in \tilde{\mu}_{\phi}^{\mathcal{B}}\left(f_{1}\right)$.

Now we show $g_{1} \vee g_{2} \in \tilde{\mu}_{\phi}^{\mathcal{B}}\left(f_{2}\right)$. Since $f_{1} \leq f_{2}$ and $\left\{g_{1}>a\right\}$ is a $\mu_{a}^{f_{1}}$-positive for all $a \in \mathbb{R}$ and for all $B \in \mathcal{B}$, we have

$$
\begin{aligned}
\int_{\left\{g_{1} \vee g_{2}>a\right\} \cap B} \underline{\phi^{\prime}}\left(f_{2}-a\right) d \mu & =\int_{\left\{g_{2}>a\right\} \cap B} \underline{\phi^{\prime}}\left(f_{2}-a\right) d \mu+\int_{\left\{g_{1}>a\right\} \cap\left\{g_{2} \leq a\right\} \cap B} \underline{\phi^{\prime}}\left(f_{2}-a\right) d \mu \\
& \geq \int_{\left\{g_{2}>a\right\} \cap B} \underline{\phi}^{\prime}\left(f_{2}-a\right) d \mu+\int_{\left\{g_{1}>a\right\} \cap\left\{g_{2} \leq a\right\} \cap B} \underline{\phi}^{\prime}\left(f_{1}-a\right) d \mu \geq 0 .
\end{aligned}
$$


Since

$$
\int_{\left\{g_{1} \vee g_{2}<a\right\} \cap B} \underline{\phi}^{\prime}\left(f_{2}-a\right) d \mu=\int_{\left\{g_{1}<a\right\} \cap\left\{g_{2}<a\right\} \cap B} \underline{\phi}^{\prime}\left(f_{2}-a\right) d \mu \leq 0,
$$

the inequalities (2.43) and (2.44) prove that $g_{1} \vee g_{2} \in \tilde{\mu}_{\phi}^{\mathcal{B}}\left(f_{2}\right)$.

As the statement (1) proves in particular that $\tilde{\mu}_{\phi}^{\mathcal{B}}(f)$ is a lattice, we will see that the set is a $\sigma$-complete lattice. Given a sequence $\left\{g_{n}\right\}_{n \in \mathbb{N}}$ in $\widetilde{\mu}_{\phi}^{\mathcal{B}}(f)$, we have that $\bigvee_{1}^{n} g_{k} \in \widetilde{\mu}_{\phi}^{\mathcal{B}}(f)$; then, from Lemma 2.11 we obtain that $\bigvee_{n \in \mathbb{N}} g_{n}=\lim _{n \rightarrow \infty} \bigvee_{1}^{n} g_{n} \in \tilde{\mu}_{\phi}^{B}(f)$. The proof $\bigwedge_{n \in \mathbb{N}} g_{n} \in \tilde{\mu}_{\phi}^{B}(f)$ is similar.

By [10, Proposition II.4.1] there exists a sequence $g_{n} \in \tilde{\mu}_{\phi}^{B}(f)$ such that inf $g_{n} \leq g \leq$ sup $g_{n}$, for every $g \in \tilde{\mu}_{\phi}^{\mathcal{B}}(f)$. Set $U_{\mathcal{B}}=\inf g_{n}$ and $V_{\mathcal{B}}=\sup g_{n}$, then $U_{B}$ and $V_{\mathcal{B}}$ are in $\tilde{\mu}_{\phi}^{\mathcal{B}}(f)$ since this set is a $\sigma$-complete lattice.

\section{Extended best $\phi$-approximation with nondecreasing functions}

When the approximation class is the monotone functions defined on $[0,1]$ we can obtain similar results as those of Theorem 2.17. Now $\Omega=[0,1], \mu$ is Lebesgue measure on the measurable sets, and $\mathcal{L}=\{(a, 1),[a, 1), \varnothing, \mathbb{R}\}_{a \in \mathbb{R}}$. Therefore, $L^{\phi^{\prime}}(\mathcal{L})$ is the set of nondecreasing functions in $L^{\phi^{\prime}}[0,1]$.

Remark 3.1. Let $g$ be a nondecreasing function on $[0,1]$. Given $a \in \mathbb{R}$, the set $\{g<a\}$ is one of the intervals $\left[0, \alpha_{a}\right)$ or $\left[0, \alpha_{a}\right]$ and similarly the set $\{g>a\}$ is $\left(\beta_{a}, 1\right]$ or $\left[\beta_{a}, 1\right]$ with $0 \leq \alpha_{a} \leq \beta_{a} \leq 1$. Then $H_{g}=\left\{a \in \mathbb{R}: \alpha_{a}=\beta_{a}\right\}$ is a dense set in $\mathbb{R}$. In fact, the complement set of $H_{g}$ is a countable set.

Note that each $C \in \mathcal{L}$ is of the form $(c, 1]$ or $[c, 1]$ and $D \in \bar{L}$ is $D=[0, d)$ or $[0, d]$. Thus

$$
\int_{\{g>a\} \cap D} \underline{\phi}^{\prime}(f-a) d \mu=\int_{\beta_{a}}^{d} \underline{\phi}^{\prime}(f-a) d \mu, \quad \int_{\{g<a\} \cap C} \underline{\phi}^{\prime}(f-a) d \mu=\int_{c}^{\alpha_{a}} \underline{\phi}^{\prime}(f-a) d \mu .
$$

Theorem 3.2. Let $L^{\phi}(\mathcal{\perp})$ be the class of the $\phi^{\prime}$-integrable nondecreasing functions in $[0,1]$. Then the following hold.

(1) The set mapping $\tilde{\mu}_{\phi}^{\mathcal{\rho}}(f)$ is a monotone operator.

(2) For every $f \in L^{\phi^{\prime}}$, the set $\tilde{\mu}_{\phi}^{\mathcal{L}}(f)$ is a $\sigma$-complete lattice.

Proof. First we prove (1), that is given $f_{1}, f_{2}$ in $L^{\phi^{\prime}}$ with $f_{1} \leq f_{2}$, for each $g_{i} \in \tilde{\mu}_{\phi}^{\mathcal{P}}\left(f_{i}\right), i=1,2$ we will see that $g_{1} \wedge g_{2} \in \tilde{\mu}_{\phi}^{\mathcal{L}}\left(f_{1}\right)$ and $g_{1} \vee g_{2} \in \tilde{\mu}_{\phi}^{\mathcal{L}}\left(f_{2}\right)$. Let $H$ be $H_{g_{1}} \cap H_{g_{2}}$, where $H_{g_{i}}$ is the set given in Remark 3.1. Recall that $g_{1} \wedge g_{2} \in \tilde{\mu}_{\phi}^{\mathcal{L}}\left(f_{1}\right)$ if and only if for each $a \in H$ and $c, d \in \mathbb{R}$

$$
\int_{\left\{g_{1} \wedge g_{2}>a\right\} \cap(0, d)} \underline{\phi}^{\prime}\left(f_{1}-a\right) d x \geq 0, \quad \int_{\left\{g_{1} \wedge g_{2}<a\right\} \cap(c, 1)} \underline{\phi}^{\prime}\left(f_{1}-a\right) d x \leq 0 .
$$

Also $g_{1} \vee g_{2} \in \tilde{\mu}_{\phi}^{\mathcal{L}}\left(f_{2}\right)$ if and only if for each $a \in H$, and $c, d \in \mathbb{R}$,

$$
\int_{\left\{g_{1} \vee g_{2}>a\right\} \cap(0, d)} \underline{\phi}^{\prime}\left(f_{2}-a\right) d x \geq 0, \quad \int_{\left\{g_{1} \vee g_{2}<a\right\} \cap(c, 1)} \underline{\phi}^{\prime}\left(f_{2}-a\right) d x \leq 0 .
$$


First we prove (3.1). Now we see that

$$
\int_{\left\{g_{1} \wedge g_{2}>a\right\} \cap(0, d)} \underline{\phi}^{\prime}\left(f_{1}-a\right) d x \geq 0,
$$

with $\left\{g_{1} \wedge g_{2}>a\right\}=\left(\beta_{1}^{a}, 1\right] \cap\left(\beta_{2}^{a}, 1\right]$. Since $\int_{\left(\beta_{1}^{a}, d\right)} \underline{\phi^{\prime}}\left(f_{1}-a\right) d x \geq 0$ to prove (3.3), we have to see that

$$
\int_{\left(\beta_{2}^{a}, d\right)} \underline{\phi^{\prime}}\left(f_{1}-a\right) d x \geq 0,
$$

where $\beta_{1}^{a} \leq \beta_{2}^{a}$. Indeed by $(\star)$, we get

$$
0 \leq \int_{\beta_{1}^{a}} a^{d} \underline{\phi}^{\prime}\left(f_{1}-a\right) d x=\int_{\beta_{1}^{a}}^{\beta_{2}^{a}} \underline{\phi^{\prime}}\left(f_{1}-a\right) d x+\int_{\beta_{2}^{a}}^{d} \underline{\phi^{\prime}}\left(f_{1}-a\right) d x .
$$

Since $\underline{\phi}^{\prime}(\cdot)$ is a nondecreasing function, we have

$$
0 \leq \int_{\beta_{1}^{a}}^{\beta_{2}^{a}} \underline{\phi^{\prime}}\left(f_{2}-a\right) d x+\int_{\beta_{2}^{a}}^{d} \underline{\phi^{\prime}}\left(f_{1}-a\right) d x .
$$

As $\int_{\beta_{1}^{a}}^{\beta_{2}^{a}} \frac{\phi^{\prime}}{N}\left(f_{2}-a\right) d x \leq 0\left(\beta_{2}^{a}=\alpha_{2}^{a}\right)$, we have $\int_{\beta_{2}^{a}}^{d} \underline{\phi^{\prime}}\left(f_{1}-a\right) d x \geq 0$, that is (3.4).

Now we will prove that

$$
\int_{\left\{g_{1} \wedge g_{2}<a\right\} \cap(c, 1)} \underline{\phi^{\prime}}\left(f_{1}-a\right) d x \leq 0 .
$$

In fact

$$
\begin{aligned}
\int_{\left\{g_{1} \wedge g_{2}<a\right\} \cap(c, 1)} \underline{\phi}^{\prime}\left(f_{1}-a\right) d x & =\int_{\left\{g_{1}<a\right\} \cap(c, 1)} \underline{\phi^{\prime}}\left(f_{1}-a\right) d x+\int_{\left\{g_{1} \geq a\right\} \cap\left\{g_{2}<a\right\} \cap(c, 1)} \underline{\phi^{\prime}}\left(f_{1}-a\right) d x \\
& \leq \int_{\left\{g_{1}<a\right\} \cap(c, 1)} \underline{\phi}^{\prime}\left(f_{1}-a\right) d x+\int_{\left\{g_{2}<a\right\} \cap\left[\left\{g_{1} \geq a\right\} \cap(c, 1)\right]} \underline{\phi^{\prime}}\left(f_{2}-a\right) d x .
\end{aligned}
$$

The last two integrals in (3.8) are less or equal than zero, so (3.7) holds. A similar argument shows that $g_{1} \vee g_{2} \in \tilde{\mu}_{\phi}^{\rho}\left(f_{2}\right)$. Therefore, the extended best $\phi$-approximation operator is a monotone operator. By $(1)$, we have that $\tilde{\mu}_{\phi}^{\mathcal{f}}(f)$ is a lattice, just setting $f=f_{1}=f_{2}$. Now by Lemma 2.11 we obtain that $\tilde{\mu}_{\phi}^{\rho}(f)$ is a $\sigma$-complete lattice.

\section{A limit theorem for extended best $\phi$-approximations}

Given a sequence $\left\{\boldsymbol{B}_{n}\right\}_{n \in \mathbb{N}}$ of $\sigma$-algebras contained in the $\sigma$-algebra $\mathcal{A}$, we consider two cases, $\boldsymbol{B}_{n} \subset \boldsymbol{B}_{n+1}$ for all $n \in \mathbb{N}$ and we set $\boldsymbol{B}_{\infty}$ for the $\sigma$-algebra generated by $\bigcup_{n} \mathcal{B}_{n}$, and if $\boldsymbol{B}_{n} \supseteq \boldsymbol{B}_{n+1}$ for all $n \in \mathbb{N}$, we set $B_{\infty}=\bigcap_{n \in \mathbb{N}} B_{n}$.

The next result is a particular case of [8, Theorem 2.8] when $\phi$ is a strictly convex function. This assumption on the function $\phi$ assures that the family of measures $\left\{\mu_{a}\right\}_{a \in \mathbb{R}}$ decreases at zero as required by Brunk and Johansen in that theorem. 
Theorem 4.1. Let $\left\{\boldsymbol{B}_{n}\right\}_{n \in \mathbb{N}}$ be an increasing or decreasing sequence of $\sigma$-algebras in $\mathbb{A}$, and let $B_{\infty}$ be the limit of the sequence. If $f \in L^{\phi^{\prime}}$, then we have for all $g_{n} \in \widetilde{\mu}_{\phi}^{B_{n}}(f), n \in \mathbb{N}$, that $\liminf \operatorname{in}_{n \rightarrow \infty} g_{n}$, and $\lim \sup _{n \rightarrow \infty} g_{n}$ are in $\tilde{\mu}_{\phi}^{B_{\infty}}(f)$.

Proof. Define $\bar{g}=\limsup _{n \rightarrow \infty} g_{n}$ and $\underline{g}=\liminf _{n \rightarrow \infty} g_{n}$, then we only prove that $\underline{g}, \bar{g} \in \tilde{\mu}_{\phi}^{B_{\infty}}(f)$, when $\left\{\boldsymbol{B}_{n}\right\}_{n \in \mathbb{N}}$ is an increasing sequence of $\sigma$-algebras, the proof for the decreasing case is similar.

First, we prove for each $f \in L^{\phi^{\prime}}$ that the set $\{\bar{g} \geq a\}$ is $\mu_{a}$-positive for all $a \in \mathbb{R}$. Let $B \in \bar{B}_{m}$, and for $H_{n}=\bigcup_{k \geq n}\left\{g_{k}>a-\epsilon_{n}\right\}$, where $\epsilon_{n}$ decreases to zero, we have that $\{\bar{g} \geq a\}=\bigcap_{n \geq 1} H_{n}$, and for all $n \in \mathbb{N}, H_{n+1} \subset H_{n}$. Now for each $n \in \mathbb{N}$, we define the following disjoint sets. For $p \geq n$, set $H_{n, n}=\left\{g_{n}>a-\epsilon_{n}\right\}, \ldots, H_{n, p}=\left\{g_{p}>a-\epsilon_{n}\right\} \cap\left\{g_{p-1} \leq a-\epsilon_{n}\right\} \cap \cdots \cap\left\{g_{n} \leq a-\epsilon_{n}\right\}$. Thus, $H_{n}=\bigcup_{p \geq n} H_{n, p}$, and then

$$
\int_{H_{n} \cap B} \underline{\phi}^{\prime}\left(f-\left(a-\epsilon_{n}\right)\right) d \mu=\sum_{p=n}^{\infty} \int_{H_{n, p} \cap B} \underline{\phi}^{\prime}\left(f-\left(a-\epsilon_{n}\right)\right) d \mu .
$$

As $\boldsymbol{B}_{n} \subset \boldsymbol{B}_{n+1}$, and for $p \geq n$, we have that $\left\{g_{p-1} \leq a-\epsilon_{n}\right\} \cap \cdots \cap\left\{g_{n} \leq a-\epsilon_{n}\right\} \in \mathcal{B}_{p}$. As $B \in \mathbb{B}_{m}$ then $B \in \mathcal{B}_{p}$ and $\int_{H_{n, p} \cap B} \underline{\phi}^{\prime}\left(f-\left(a-\epsilon_{n}\right)\right) d \mu \geq 0$ for $m \leq n \leq p$. Thus $\int_{H_{n} \cap B} \underline{\phi^{\prime}}\left(f-\left(a-\epsilon_{n}\right)\right) d \mu \geq 0$, and by Lebesgue's theorem, we get

$$
\int_{\{\bar{g} \geq a\} \cap B} \underline{\phi^{\prime}}(f-a) d \mu=\lim _{n \rightarrow \infty} \int_{H_{n} \cap B} \underline{\phi^{\prime}}\left(f-\left(a-\epsilon_{n}\right)\right) d \mu \geq 0
$$

for all $B \in \bigcup_{n \in \mathbb{N}} B_{n}$. Now we have (4.2) for all $B \in B_{\infty}$. In fact, the set $D=\{B \in \mathcal{A}$ : $\left.\int_{\{\bar{g} \geq a\} \cap B} \underline{\phi^{\prime}}(f-a) d \mu \geq 0\right\}$ is a monotone class, that is, the set $D$ is closed for increasing and decreasing sequences of sets. As $\bigcup_{n \in \mathbb{N}} B_{n} \subset D$ and this union is an algebra of sets, the monotone class generated by it is $B_{\infty}$, that is, $B_{\infty} \subset D$.

Now let us prove that the set $\{\bar{g} \leq a\}$ is $\mu_{a}$-negative for all $a \in \mathbb{R}$. As $\{\bar{g} \leq a\}=\bigcap_{n \in \mathbb{N}}\{\bar{g}<$ $a+1 / n\}$ and

$$
\int_{\{\bar{g} \leq a\} \cap B} \underline{\phi}^{\prime}(f-a) d \mu=\lim _{n \rightarrow \infty} \int_{\{\bar{g}<a+1 / n\} \cap B} \underline{\phi}^{\prime}(f-a) d \mu,
$$

then we have to prove that for all $a \in \mathbb{R}$, the set $\{\bar{g}<a\}$ is $\mu_{a}$-negative. Since $\{\bar{g}<a\}=$ $\bigcup_{n \geq 1} \bigcap_{k \geq n}\left\{g_{k}<a-\epsilon_{n}\right\}=\bigcup_{n \geq 1} H_{n}$, where $H_{n}=\bigcap_{k \geq n}\left\{g_{k}<a-\epsilon_{n}\right\}$ and $\epsilon_{n} \searrow 0$, we have for all $n \in \mathbb{N}$ that $H_{n} \subset H_{n+1}$. Then

$$
\int_{\{\bar{g}<a\} \cap B} \frac{\phi^{\prime}}{}(f-a) d \mu=\lim _{n \rightarrow \infty} \int_{H_{n} \cap B} \underline{\phi}^{\prime}\left(f-\left(a-\epsilon_{n}\right)\right) d \mu,
$$

for a fixed set $B \in B_{m}$.

Set $G_{n}=\bigcap_{k \geq n+1}\left\{g_{k}<a-\epsilon_{n}\right\}$ and note that $H_{n}=G_{n} \cap\left\{g_{n}<a-\epsilon_{n}\right\}$. Then for $m \leq n$, we have

$$
\begin{gathered}
\int_{\left\{g_{n}<a-\epsilon_{n}\right\} \cap B \cap G_{n}} \underline{\phi^{\prime}}\left(f-\left(a-\epsilon_{n}\right)\right) d \mu+\int_{\left\{g_{n}<a-\epsilon_{n}\right\} \cap B \cap G_{n}^{c}} \underline{\phi}^{\prime}\left(f-\left(a-\epsilon_{n}\right)\right) d \mu \\
=\int_{\left\{g_{n}<a-\epsilon_{n}\right\} \cap B} \underline{\phi}^{\prime}\left(f-\left(a-\epsilon_{n}\right)\right) d \mu \leq 0 .
\end{gathered}
$$


Now, we prove the following inequality:

$$
\int_{\left\{g_{n}<a-\epsilon_{n}\right\} \cap B \cap G_{n}^{c}} \underline{\phi}^{\prime}\left(f-\left(a-\epsilon_{n}\right)\right) d \mu \geq 0 .
$$

We can see that

$$
\left\{g_{n}<a-\epsilon_{n}\right\} \cap B \cap G_{n}^{c}=\bigcup_{k \geq n+1} A_{k}
$$

where $A_{k}$ are the following disjoint sets

$$
\begin{aligned}
A_{n+1} & =\left\{g_{n+1} \geq a-\epsilon_{n}\right\} \cap B \cap\left\{g_{n}<a-\epsilon_{n}\right\}, \\
& \vdots \\
A_{k} & =\left\{g_{k} \geq a-\epsilon_{n}\right\} \cap \bigcap_{i=n+1}^{k-1}\left\{g_{i}<a-\epsilon_{n}\right\} \cap\left\{g_{n}<a-\epsilon_{n}\right\} \cap B .
\end{aligned}
$$

Then

$$
\int_{\left\{g_{n}<a-\epsilon_{n}\right\} \cap B \cap G_{n}^{c}} \underline{\phi}^{\prime}\left(f-\left(a-\epsilon_{n}\right)\right) d \mu=\sum_{k \geq n+1} \int_{A_{k}} \underline{\phi}^{\prime}\left(f-\left(a-\epsilon_{n}\right)\right) d \mu .
$$

Since $A_{k}=\left\{g_{k} \geq a-\epsilon_{n}\right\} \cap B_{k}$, where $B_{k} \in B_{k}$, we have (4.6). Therefore by (4.5), we have

$$
\int_{H_{n} \cap B} \underline{\phi^{\prime}}\left(f-\left(a+\epsilon_{n}\right)\right) d \mu \leq 0
$$

for all $B \in \bigcup_{n \in \mathbb{N}} 乃_{n}$. Thus by (4.10), (4.4), and (4.3), we get

$$
\int_{\{\bar{g} \leq a\} \cap B} \underline{\phi^{\prime}}(f-a) d \mu \leq 0
$$

for all $B \in \bigcup_{n \in \mathbb{N}} \mathcal{B}_{n}$. Therefore, the result is satisfied for all $B \in \mathbb{B}_{\infty}$. Thus $\bar{g} \in \widetilde{\mu}_{\phi}^{B_{\infty}}(f)$.

We have $\{\underline{g} \leq a\}=\bigcap_{n \geq 1} H_{n}$, where $H_{n}=\bigcup_{k \geq n}\left\{g_{k}<a+\epsilon_{n}\right\}$ and $\epsilon_{n} \searrow 0$, then $H_{n+1} \subset H_{n}$ for all $n \in \mathbb{N}$. Since $f \in L^{\phi^{\prime}}$ we have for all $B \in B_{\infty}$ that

$$
\int_{\{\underline{g} \leq a\} \cap B} \underline{\phi}^{\prime}(f-a) d \mu=\lim _{n \rightarrow \infty} \int_{H_{n} \cap B} \underline{\phi}^{\prime}\left(f-\left(a+\epsilon_{n}\right)\right) d \mu .
$$

For $p>n$ define the following disjoint sets $H_{n, n}=\left\{g_{n}<a+\epsilon_{n}\right\}$ and $H_{n, p}=\left\{g_{p}<a+\epsilon_{n}\right\} \cap\left\{g_{p-1} \geq\right.$ $\left.a+\epsilon_{n}\right\} \cap \cdots \cap\left\{g_{n} \geq a+\epsilon_{n}\right\}$. Then for $B \in \mathcal{B}_{m}$ we have

$$
\int_{H_{n} \cap B} \underline{\phi^{\prime}}\left(f-\left(a+\epsilon_{n}\right)\right) d \mu=\sum_{p \geq n} \int_{H_{n, p} \cap B} \underline{\phi^{\prime}}\left(f-\left(a+\epsilon_{n}\right)\right) d \mu .
$$


Now if $m \leq n \leq p, H_{n, p} \cap B=\left\{g_{k}<a+\epsilon_{n}\right\} \cap B^{\star}$, where $B^{\star} \in \mathcal{B}_{p}$. Thus

$$
\int_{H_{n, p} \cap B} \underline{\phi^{\prime}}\left(f-\left(a+\epsilon_{n}\right)\right) d \mu \leq 0 .
$$

Then by (4.12) and (4.14), we have for all $B \in \bigcup_{n \in \mathbb{N}} 乃_{n}$ that

$$
\int_{\{\underline{g} \leq a\} \cap B} \underline{\phi}^{\prime}(f-a) d \mu \leq 0 .
$$

Therefore, we have (4.15) for all $B \in B_{\infty}$.

Let us see now that

$$
\int_{\{\underline{g} \geq a\} \cap B} \underline{\phi}^{\prime}(f-a) d \mu \geq 0
$$

for all $B \in \bigcup_{n \in \mathbb{N}} B_{n}$.

As $\{g \geq a\}=\bigcap_{n \in \mathbb{N}}\{g>a-1 / n\}$, we have to prove that for all $a \in \mathbb{R}$, the set $\{g>a\}$ is $\mu_{a}$-positive. We have that $\{g>a\}=\bigcup_{n \geq 1} \bigcap_{k \geq n}\left\{g_{k} \geq a+\epsilon_{n}\right\}=\bigcup_{n \geq 1} H_{n}$, where $H_{n}$ is the increasing sequence $H_{n}=\bigcap_{k \geq n}\left\{g_{k} \geq a+\epsilon_{n}\right\}$ and $\epsilon_{n} \searrow 0$. Then we have

$$
\int_{\{\underline{g}>a\} \cap B} \underline{\phi^{\prime}}(f-a) d \mu=\lim _{n \rightarrow \infty} \int_{H_{n} \cap B} \underline{\phi}^{\prime}\left(f-\left(a+\epsilon_{n}\right)\right) d \mu .
$$

Set $G_{n}=\bigcap_{k \geq n+1}\left\{g_{k} \geq a+\epsilon_{n}\right\}$ and note that $H_{n}=G_{n} \cap\left\{g_{n} \geq a+\epsilon_{n}\right\}$. Then for $B \in \mathcal{B}_{m}, m \leq n$, we have

$$
\begin{aligned}
0 & \leq \int_{\left\{g_{n} \geq a+\epsilon_{n}\right\} \cap B} \underline{\phi^{\prime}}\left(f-\left(a+\epsilon_{n}\right)\right) d \mu \\
& =\int_{\left\{g_{n} \geq a+\epsilon_{n}\right\} \cap B \cap G_{n}} \underline{\phi}^{\prime}\left(f-\left(a+\epsilon_{n}\right)\right) d \mu+\int_{\left\{g_{n} \geq a+\epsilon_{n}\right\} \cap B \cap G_{n}^{c}} \phi^{\prime}\left(f-\left(a+\epsilon_{n}\right)\right) d \mu .
\end{aligned}
$$

Now, we prove

$$
\int_{\left\{g_{n} \geq a+\epsilon_{n}\right\} \cap B \cap G_{n}^{c}} \underline{\phi}^{\prime}\left(f-\left(a+\epsilon_{n}\right)\right) d \mu \leq 0 .
$$

We can see that

$$
\left\{g_{n} \geq a+\epsilon_{n}\right\} \cap B \cap G_{n}^{c}=\bigcup_{k \geq n+1} A_{k}
$$

where $A_{k}$ are the following disjoint sets:

$$
\begin{aligned}
A_{n+1} & =\left\{g_{n+1}<a+\epsilon_{n}\right\} \cap B \cap\left\{g_{n} \geq a+\epsilon_{n}\right\}, \\
& \vdots \\
A_{k} & =\left\{g_{k}<a+\epsilon_{n}\right\} \cap \bigcap_{i=n+1}^{k-1}\left\{g_{i} \geq a+\epsilon_{n}\right\} \cap\left\{g_{n} \geq a+\epsilon_{n}\right\} \cap B .
\end{aligned}
$$


Then

$$
\int_{\left\{g_{n} \geq a+\epsilon_{n}\right\} \cap B \cap G_{n}^{c}} \underline{\phi}^{\prime}\left(f-\left(a+\epsilon_{n}\right)\right) d \mu=\sum_{k \geq n+1} \int_{A_{k}} \underline{\phi}^{\prime}\left(f-\left(a+\epsilon_{n}\right)\right) d \mu .
$$

Since $A_{k}=\left\{g_{k}<a+\epsilon_{n}\right\} \cap B_{k}$, where $B_{k} \in B_{k}$, we have (4.19). Therefore by (4.18), we have

$$
\int_{H_{n} \cap B} \underline{\phi^{\prime}}\left(f-\left(a+\epsilon_{n}\right)\right) d \mu \geq 0
$$

for all $B \in \bigcup_{n \in \mathbb{N}} \beta_{n}$. Thus by (4.23) and (4.17), we get

$$
\int_{\{\underline{g} \geq a\} \cap B} \underline{\phi}^{\prime}(f-a) d \mu \geq 0
$$

for all $B \in \bigcup_{n \in \mathbb{N}} B_{n}$. Therefore, the result is satisfied for all $B \in \mathbb{B}_{\infty}$. Thus $\underline{g} \in \tilde{\mu}_{\phi}^{B_{\infty}}(f)$.

\section{Acknowledgment}

This work was supported by CONICET and UNSL grants.

\section{References}

[1] D. Landers and L. Rogge, "Best approximants in $L_{\phi}$-spaces," Zeitschrift für Wahrscheinlichkeitstheorie und Verwandte Gebiete, vol. 51, no. 2, pp. 215-237, 1980.

[2] D. Landers and L. Rogge, "Isotonic approximation in $L_{s}$," Journal of Approximation Theory, vol. 31, no. 3, pp. 199-223, 1981.

[3] F. Mazzone and H. Cuenya, "Isotonic approximations in $L_{1}$," Journal of Approximation Theory, vol. 117, no. 2, pp. 279-300, 2002.

[4] S. Favier and F. Zo, "Extension of the best approximation operator in Orlicz spaces and weak-type inequalities," Abstract and Applied Analysis, vol. 6, no. 2, pp. 101-114, 2001.

[5] S. Favier and F. Z6, "A Lebesgue type differentiation theorem for best approximations by constants in Orlicz spaces," Real Analysis Exchange, vol. 30, no. 1, pp. 29-42, 2005.

[6] S. Favier and F. Z6, "Sharp conditions for maximal inequalities of the best approximation operator," preprint.

[7] F. Mazzone and H. Cuenya, "Maximal inequalities and Lebesgue's differentiation theorem for best approximant by constant over balls," Journal of Approximation Theory, vol. 110, no. 2, pp. 171-179, 2001.

[8] H. D. Brunk and S. Johansen, "A generalized Radon-Nikodym derivative," Pacific Journal of Mathematics, vol. 34, pp. 585-617, 1970.

[9] F. Mazzone and H. Cuenya, "A characterization of best $\phi$-approximants with applications to multidimensional isotonic approximation," Constructive Approximation, vol. 21, no. 2, pp. 207-223, 2005.

[10] J. Neveu, Mathematical Foundations of the Calculus of Probability, Holden-Day, San Francisco, Calif, USA, 1965. 


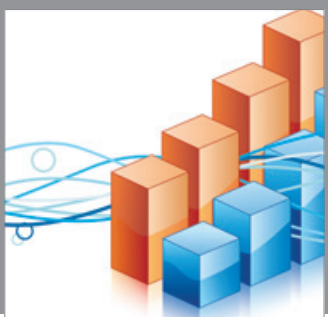

Advances in

Operations Research

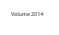

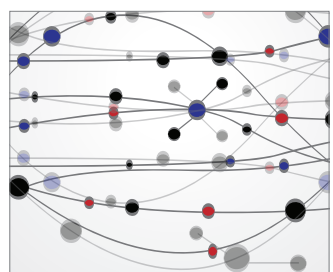

\section{The Scientific} World Journal
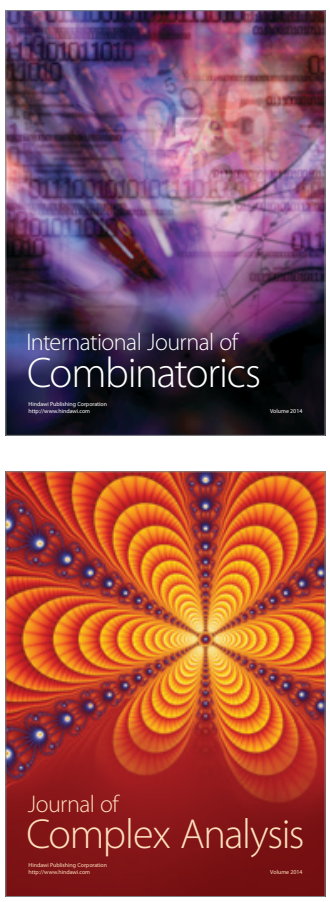

International Journal of

Mathematics and

Mathematical

Sciences
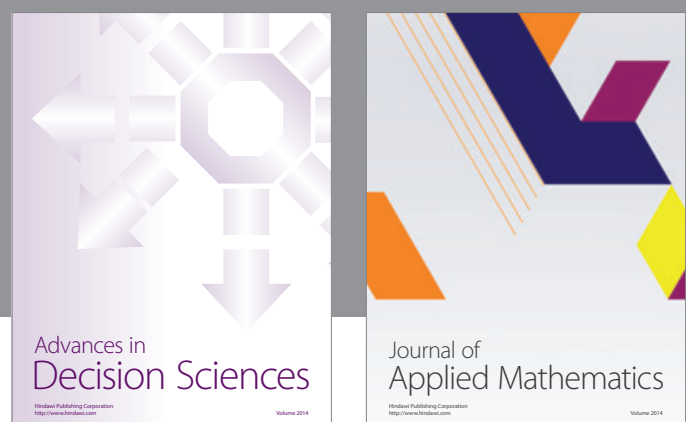

Journal of

Applied Mathematics
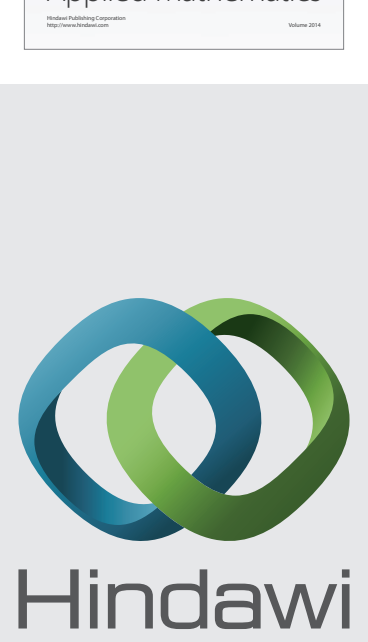

Submit your manuscripts at http://www.hindawi.com
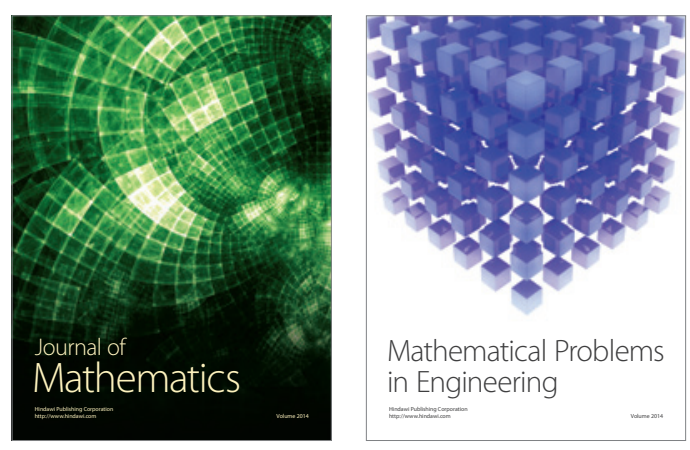

Mathematical Problems in Engineering
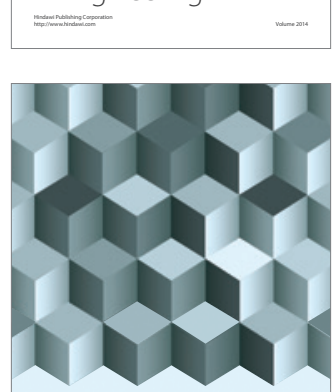

Journal of

Function Spaces
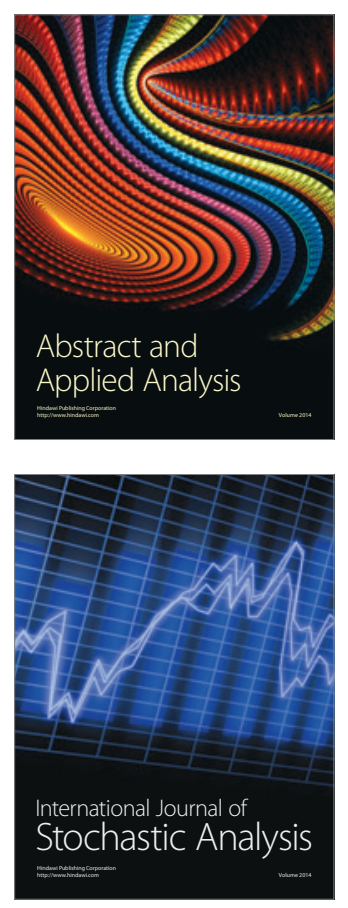

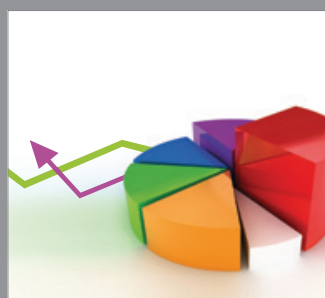

ournal of

Probability and Statistics

Promensencen
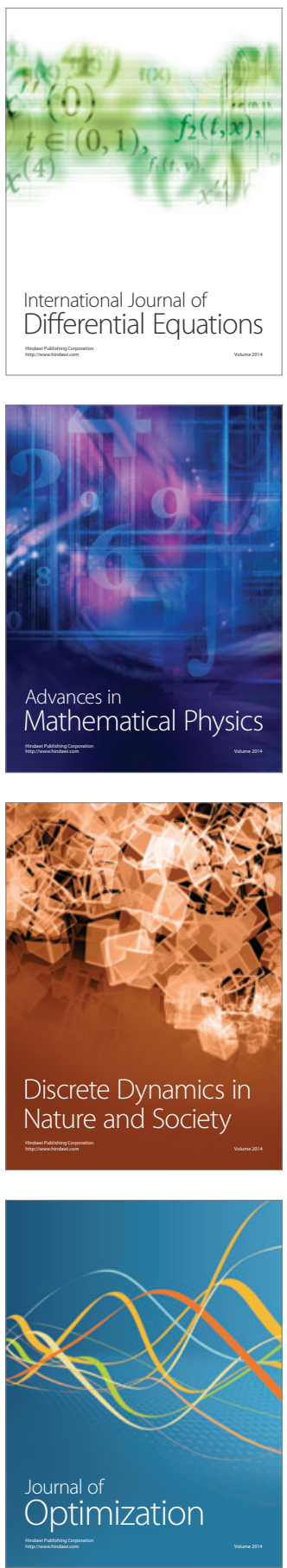